\title{
Documentation of medicinal plants used by Aneuk Jamee tribe in Kota Bahagia Sub-district, South Aceh, Indonesia
}

\author{
ADI BEJO SUWARDI ${ }^{1, \boldsymbol{v}}$, MARDUDI ${ }^{1}$, ZIDNI ILMAN NAVIA $^{2}$, BAIHAQI $^{3}$, MUNTAHA $^{4}$ \\ ${ }^{1}$ Department of Biology Education, Faculty of Teacher Training and Education, Universitas Samudra. Jl. Prof. Dr. Syarief Thayeb, Meurandeh, Langsa \\ 24354, Aceh, Indonesia. Tel.: +62-641-426535, `email: adi.bsw@gmail.com \\ ${ }^{2}$ Department of Biology, Faculty of Engineering, Universitas Samudra. Jl. Prof. Dr. Syarief Thayeb, Meurandeh, Langsa 24354, Aceh, Indonesia \\ ${ }^{3}$ Department of English Education, Faculty of Teacher Training and Education, Universitas Samudra. Jl. Prof. Dr. Syarief Thayeb, Meurandeh, Langsa \\ 24354, Aceh, Indonesia \\ ${ }^{4}$ Sekolah Tinggi Ilmu Ekonomi Boedi Oetomo. Jl. Ampera, Kota Baru, Pontianak 78121, West Kalimantan, Indonesia
}

Manuscript received: 6 November 2020. Revision accepted: 6 December 2020.

\begin{abstract}
Suwardi AB, Mardudi, Navia ZI, Baihaqi, Muntaha. 2021. Documentation of medicinal plants used by Aneuk Jamee tribe in Kota Bahagia sub-district, South Aceh, Indonesia. Biodiversitas 21: 6-15. Aneuk Jamee is one of the ethnic communities living along the western-south coast of Aceh. Various plants are used by the Aneuk Jamee tribe as a traditional medicine in treating diseases and disorders. The aim of this study was therefore to document the medicinal plants used by the Aneuk Jamee tribe in the Kota Bahagia subdistrict, South Aceh, Indonesia. This study was conducted in three villages, namely Jombo Keupok, Seuneubok Kuranji, and Alur Dua Mas, Kota Bahagia subdistrict, South Aceh District, Aceh Province. This study was based on field surveys, plant collection, and interviews with the local people. Interviews were performed with 60 informants selected by using the Snowball Sampling technique. A total of 96 medicinal plant species, consisting of 50 families, have been documented to be used by the Aneuk Jamee tribe in the Kota Bahagia subdistrict. Fifty-nine (61\%) species are cultivated and 37 (39\%) species are wild. Leaves are the most widely used plant part $(28 \%)$, followed by the fruit $(19 \%)$, flower and tuber $(6 \%$ each), seed $(3 \%)$, and sap $(2 \%)$ and the main mode of preparations are decoction $(60 \%)$, followed by raw consumption (14\%), smeared (10\%), pounded (7\%), dropped (6\%), and affixed and squeezed (1\% each). The high informant consensus factor ( $\mathrm{ICF}=0.98$ ) was assigned to the diseases of the blood and blood-forming organs and certain disorders involving the immune mechanism category.
\end{abstract}

Keywords: Biodiversity, Traditional medicine, Aneuk Jamee, Kota Bahagia

\section{INTRODUCTION}

Plants are valuable in human livelihoods, providing a source of nourishment (Navia and Chikmawati 2015; Elfrida et al. 2010; Navia et al. 2019; Navia et al. 2020a; Suwardi et al. 2020a; Suwardi et al. 2020b), condiments and spices (Navia et al. 2020b), fragrances (Dar et al. 2017), ritual or magical values (Abbink 1995; Sutrisno et al. 2020), and traditional medicine (Liu et al. 2009; Silalahi et al. 2015; Nurlinda et al. 2018; Tsioutsiou et al. 2019; Suwardi et al. 2019; Gowramma et al. 2020). Indonesia comprises more than 40,000 species of plants, of which approximately 6,000 have been used in traditional healing processes (Elfahmi et al. 2014). These plants are considered for their richness in biologically active secondary metabolites and essential oils for disease prevention (Ngbolua et al. 2018; Suwardi et al. 2018; Ortiz et al. 2020), and human beings have refined medicinal plants and their chemical properties in varying ways for therapeutic usages (Colalto 2018; Kumar et al. 2018). Traditional medicine has been focused on meeting the objectives of wider coverage of primary healthcare provision across all countries of the world (Bekalo et al. 2009). The World Health Organization (WHO) has confirmed that about $80 \%$ of the people living in developing countries rely on medicinal plants as part of their healthcare system (Ngbolua et al. 2016). The relationship between humans and plants has long been identified as one of the aspects of human civilization, particularly in medicinal domains (Yeung et al. 2020).

Aneuk Jamee is one of the ethnic communities in Indonesia inhabiting along the west-south coast of Aceh (Melalatoa 1995). Historical evidence suggests that the Minang tribe from West Sumatra migrated to western Aceh in the 17th century and assimilated with the indigenous population to establish new customs and culture recognized as Aneuk Jamee (Ramli and Erwandi 2019). Like most tribes in the province of Aceh, Aneuk Jamee also uses various species of plants as traditional medicines. This knowledge is gained through experience passed down from generation to generation. However, the knowledge of medicinal plants possessed by rural communities has rarely been documented and is generally known merely to the elderly or traditional healers. Moreover, the younger generation, especially those already integrated into modern life, is less concerned with this traditional knowledge (Maulidiah et al. 2020). Several previous studies have shown the lack of ability of older generations to pass traditional knowledge to the younger generation (Sousa et al. 2012; Saynez-Vaquest et al. 2016; Navia et al. 2020a), resulting in the disaffection of younger generations from their surrounding environment and the ultimate loss of 
nature-related information. Traditional knowledge and use of medicinal plants are an integrated component among the Aneuk Jamee tribe, the extent of which has not yet been extensively studied. Documentation of medicinal uses of plants across ethnobotanical studies is important as an opportunity to promote the development of pharmaceutical drugs and the conservation of plants (Calzada and Bautista 2020). Various ethnobotanical studies suggest the use of medicinal plants for the treatment that has been used in their respective populations over several generations (Navia et al. 2020a; Navia et al. 2020b; Sutrisno et al. 2020; Suwardi et al. 2020c). The aim of this study was therefore to document the medicinal plants used by the Aneuk Jamee tribe in the Kota Bahagia sub-district, South Aceh, Indonesia.

\section{MATERIALS AND METHODS}

\section{Study area}

South Aceh district is located between 02 $23^{\prime} 24^{\prime \prime}$ $03^{\circ} 44^{\prime} 24^{\prime \prime} \mathrm{N}$ and $96^{\circ} 57^{\prime} 36^{\prime \prime}-97^{\circ} 56^{\prime} 24^{\prime \prime} \mathrm{E}$ with an average elevation of 25 meters above sea level (masl). This district has an area of 4,173,67 $\mathrm{km}^{2}$ with land use is dominated by protected areas, namely protected forests (36.5\%), Gunung Leuser National Park (18.8\%), Trumon Wildlife Reserve (13.9\%), Animal Corridors (0.2\%), Coastal Border (0.3\%) and River Border $(1.2 \%)$ of the total area. South Aceh district consists of 18 sub-districts and 260 villages with a total population of $238,081,000$ people (The Central Bureau of Statistics of South Aceh District 2020).

Kota Bahagia is one of the subdistricts in the South Aceh district located between $3^{\circ} 0^{\prime} 46^{\prime \prime} \mathrm{N}, 9^{\circ} 33^{\prime} 30^{\prime \prime} \mathrm{E}, 0.6-$ 44.2 masl. Climatic conditions in the Kota Bahagia subdistrict are tropical humid, having an average annual rainfall varying $161.33 \mathrm{~mm}-440.78 \mathrm{~mm}$, and the average daily temperature of the area is $29^{\circ} \mathrm{C}$. This subdistrict has an area of $195.82 \mathrm{~km}^{2}$ with a total population of 7,266 people consisting of 3,580 men and 3,686 women (The Central Bureau of Statistics of South Aceh District 2020). The study was conducted from July to Sept 2020 in the Jombo Keupok, Seuneubok Kuranji, and Alur Dua Mas villages, Kota Bahagia subdistrict, South Aceh District, Aceh Province (Figure 1).

\section{Data collection}

Data were collected through in-depth interviews with 60 informants who were selected using the Snowball Sampling technique (Table 1). Key people are people who practice the use of plants for traditional medicines. Subsequent informants were determined by the direction of the previous respondents. The interview activities have been carried by using a semi-structured questionnaire to assess traditional practices for the use of medicinal plant species, the part used, and the mode of preparation.

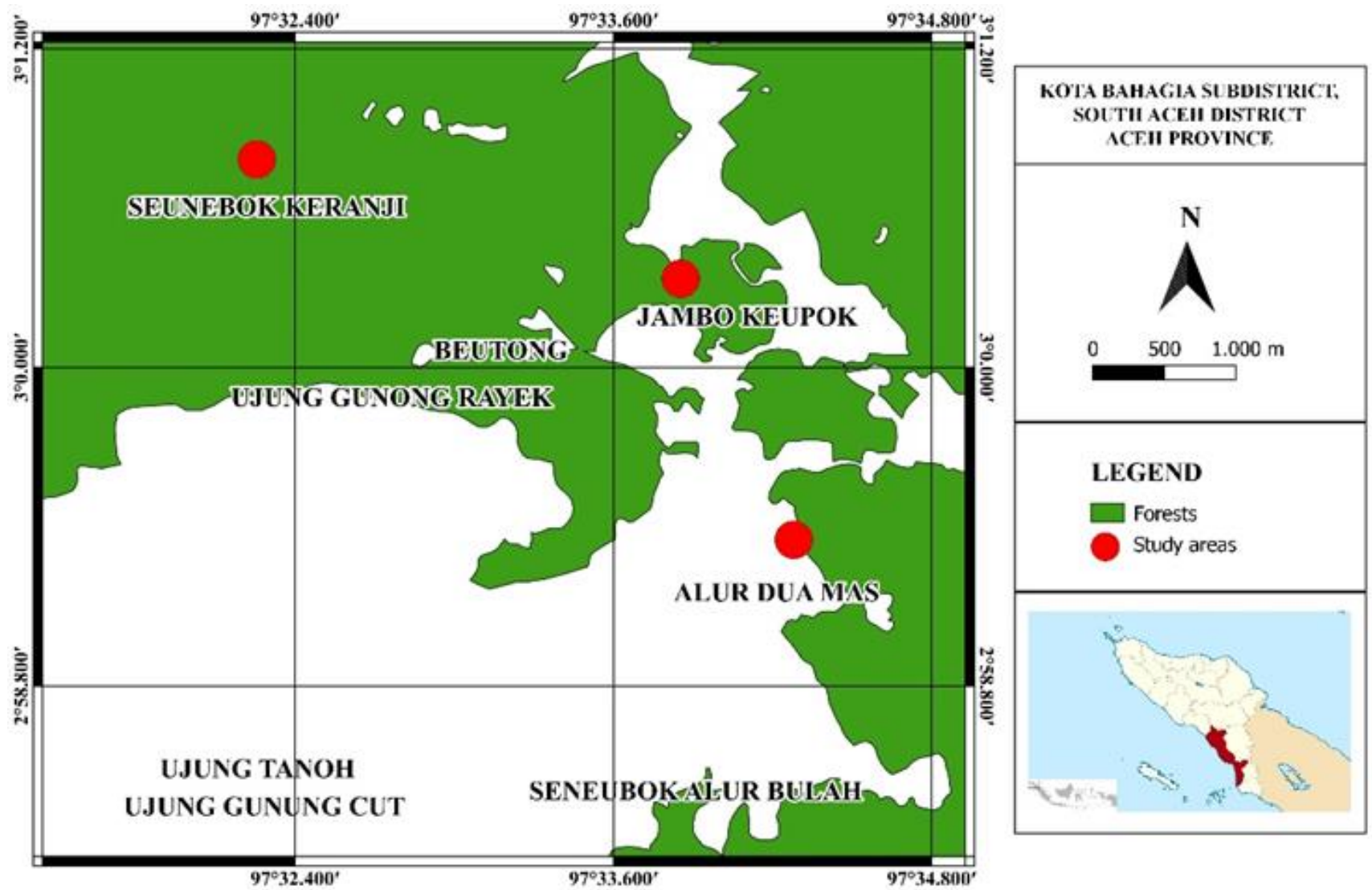

Figure 1. Map of Kota Bahagia Subdistrict, South Aceh District, Aceh Province, Indonesia showing the study area 
Table 1. The demographic structure of respondents

\begin{tabular}{llrr}
\hline Parameter & Specification & Frequency & Percentage \\
\hline Gender & Male & 38 & 63.3 \\
& Female & 22 & 36.7 \\
Age & $15-25$ & 5 & 8.3 \\
& $26-35$ & 12 & 20 \\
& $36-45$ & 15 & 25 \\
& $46-55$ & 14 & 23.3 \\
& $56-65$ & 10 & 16.7 \\
Education & $>65$ & 4 & 6.7 \\
& None & 6 & 10 \\
& Elementary School & 17 & 28.3 \\
& Junior High School & 16 & 26.7 \\
& Senior High School & 16 & 26.7 \\
& University & 5 & 8.3 \\
\hline
\end{tabular}

The samples of plants were collected along with noting down their vernacular names. The identification of plant species was conducted in the Laboratory of Biology, Samudra University, Aceh, Indonesia. The botanical names have been updated using the Plants of the World online. (http: //www.plantsoftheworldonline.org). For this study, we do not deposit plant specimens into the herbarium.

\section{Data analysis}

Use Value (UV)

The Use Value is calculated as the proportion of the number of citations per species (U) to the number of informants $(\mathrm{N})$ following Polat et al. (2015):

$$
\mathrm{UV}=\mathrm{U} / \mathrm{N}
$$

High UV implies high use-reports for a plant that possesses significance to the local community. Low UV suggests that there are few reports related to its use.

\section{Informant Consensus Factor (ICF)}

Informant Consensus Factor (ICF) is determined by using the following Cornara et al. (2014):

$$
\mathrm{ICF}=(\mathrm{Nur}-\mathrm{Nt}) / \mathrm{Nur}-1
$$

Where Nur is the number of useful reports in each category and $\mathrm{Nt}$ is the number of species used by all informants for a particular category.

\section{RESULTS AND DISCUSSION}

\section{Characteristics of medicinal plants}

A total of 96 medicinal plant species, consisting of 50 families, have been documented which are used by the Aneuk Jamee tribe in the Kota Bahagia subdistrict (Table 2).

Lamiaceae and Fabaceae are the most represented plant family with 7 species, followed by Asteraceae (6 species), Euphorbiaceae (5 species), and Acanthaceae, Amaranthaceae, Arecaceae, Rubiaceae, and Solanaceae with 4 species each. The other 41 families possess one to 3 representative species each. Fifty-nine (61.46\%) species are cultivated and 37 (38.54\%) species are wild. Piper betle and Psidium guajava are common plant species that have been used as traditional medicine by local people in the study area. $P$. betle widely found planted in home gardens and consistent with the reported in West Java, Central Java, East Java, and Bali (Sari et al. 2015). Respondents stated that along with the use as medicine, $P$. betle is also used as a material in traditional ceremonies. In addition, $P$. guajava provides multiple purposes, along with that plant as a medicine, this plant harvested the fruit for raw consumption. P. guajava has widely grown in the home garden, in line with the report by Elfrida et al. (2020) in the Aceh Tamiang district. The average number of species identified by each age group of the respondent ranged from $12.8 \pm 0.11$ ( $15-25$ years) to $94.81 \pm 0.02$ (> 65 years $)$. In addition, the average number of species identified by each educational status of the respondent ranged from $22.8 \pm$ 1.14 (Senior High School) to $42.22 \pm 0.12$ (Junior High School). Thirty-seven (38.5\%) medicinal plants were recognized by all respondents include Durio zibethinus, Myristica fragrans, P. betle, P. guajava, and Kaempferia galanga.

\section{Plant part used}

The leaves $(28 \%)$ were the most used as traditional medicine, followed by the fruit (19\%), flower and tuber (6\% each), seed (3\%), and sap (2\%). Certain diseases are treated with the whole plant, approximately $20 \%$ of the total plant species identified in this study area (Figure 2).

Leaves are the most commonly used plant part as an ethnomedicinal practice of the Aneuk Jamee tribe. The leaves have been widely used in traditional medicine due to the presence of bioactive compounds other than parts of plants (Ismail and Ahmad 2019). These studies are consistent with the reports by Tantengco et al. (2018) that local communities in the Philippines have the most used leaves in ethnomedicinal practices compare to other parts of the plants. During the discussion, the respondents stated that leaves are also easy to collect and are the most abundant part of the plant. In addition, the use of plant parts as traditional medicine can protect plants and ensure the sustainability of plant usage. Leaves are known to synthesis a wide range of secondary metabolites such as alkaloids, saponins, and phenolic compounds (Ghorbani 2005; Tantengco et al. 2018) that could be concerned for the pharmacological effects encountered by the Aneuk Jamee tribe. Several of the plants used as traditional medicine by the Aneuk Jamee tribe, such as Psidium guajava, are known to have flavonoids and tannins expressing antidiarrheal activity (Ezekwesili et al. 2010; Shakeera et al. 2013). In addition, secondary metabolites such as flavonoids, tannins, glycosides, and terpenoids, revealed by P.guajava leaves extract, have been reported to have various pharmacological properties such as antibacterial, anticough, antidiabetic, antihyperlipidemic, cardioprotective, antimutagenic, hepatoprotective, and larvicidal (Ngbolua et al. 2018). 
Table 2. Utilization of plant species as traditional medicine by Aneuk Jamee tribe in Kota Bahagia Sub-district, South Aceh, Indonesia

\begin{tabular}{|c|c|c|c|c|c|c|c|c|}
\hline Scientific name & Family & $\begin{array}{l}\text { Vernacular } \\
\text { name }\end{array}$ & $\begin{array}{l}\text { Plant } \\
\text { type }\end{array}$ & Habit & Part used & Mode of preparation & Disease & $\mathbf{U V}$ \\
\hline Acalypha australis $\mathrm{L}$. & Euphorbiaceae & Anting-anting & Shrub & Wild & Whole plants & Decoction, smeared & Wounds, diarrhoea, dysentery, cough & 0.30 \\
\hline Acalypha hispida Burm.f. & Euphorbiaceae & Ekor kucing & Shrub & Cultivated & Leaves, flower & $\begin{array}{l}\text { Decoction, pounded, } \\
\text { smeared }\end{array}$ & $\begin{array}{l}\text { Dysentery, nosebleed, anthelmintic, skin } \\
\text { burn }\end{array}$ & 0.68 \\
\hline Acanthus ebracteatus Vahl & Acanthaceae & Jeruju & Herb & Wild & Leaves & Decoction & Hepatitis & 0.30 \\
\hline Achyranthes aspera $\mathrm{L}$. & Amaranthaceae & Bungo bayom & Herb & Cultivated & Leaves, flower & Decoction & Fever, malaria, rheumatism, dysmenorrhea & 0.80 \\
\hline Adenanthera pavonina $\mathrm{L}$. & Fabaceae & Si bayi & Tree & Wild & Leaves & Decoction & Gastric ulcer & 0.53 \\
\hline Ageratum conyzoides $\mathrm{L}$. & Asteraceae & Simamih & Herb & Wild & Leaves & Smeared, decoction & Sore throat, wounds, itch & 0.47 \\
\hline Alpinia galanga $(\mathrm{L}$.$) Willd.$ & Zingiberaceae & Langkuweh & Herb & Cultivated & Tubers & Decoction & Cough, fever, flatulence, stomachache & 0.97 \\
\hline Amaranthus spinosus L. & Amaranthaceae & Bayam duri & Herb & Cultivated & Whole plants & Decoction, smeared & Fever, diarrhoea & 0.68 \\
\hline Annona muricata $\mathrm{L}$. & Annonaceae & Dienlando & Tree & Cultivated & Fruit & Raw consumption & Gastric ulcer, sprue, hypertension & 0.97 \\
\hline Areca саtechu L. & Arecaceae & Pinang & Palm & Cultivated & Seed & Raw consumption & Gastric ulcer, jaundice, itch & 0.95 \\
\hline Arenga pinnata (Wurmb) Merr. & Arecaceae & Ijuok & Palm & Wild & Fruit & Decoction & Diabetes & 0.53 \\
\hline Artemisia vulgaris $\mathrm{L}$ & Asteraceae & Barucina & Herb & Wild & Leaves & Decoction & Dysentery, diarrhea, leukorrhea & 0.30 \\
\hline Averrhoa bilimbi $\mathrm{L}$. & Oxalidaceae & Limbieng & Tree & Cultivated & Fruit & Raw consumption & Hypertension & 0.53 \\
\hline Barleria cristata L. & Acanthaceae & Daun madu & Herb & Cultivated & Whole plants & $\begin{array}{l}\text { Decoction, pounded, } \\
\text { smeared }\end{array}$ & Gastric ulcer, pimple & 0.37 \\
\hline Basella alba $\mathrm{L}$. & Basellaceae & Limayuong & Herb & Cultivated & Leaves, fruit & $\begin{array}{l}\text { Decoction, pounded, } \\
\text { smeared }\end{array}$ & $\begin{array}{l}\text { Gastric ulcer, toothache, insomnia, skin } \\
\text { burn }\end{array}$ & 0.48 \\
\hline Bidens pilosa $\mathrm{L}$. & Asteraceae & Bungo adet-adet & Herb & Wild & Whole plants & Decoction & Tuberculosis, haematemesis & 0.43 \\
\hline Canna indica $\mathrm{L}$. & Cannaceae & Bunga tasbih & Herb & Cultivated & Leaves, flowers & Decoction & Hypertension, fever, jaundice & 0.52 \\
\hline Carica papaya $\mathrm{L}$. & Caricaceae & Botiek & Shrub & Cultivated & Leaves & $\begin{array}{l}\text { Raw consumption, } \\
\text { decoction }\end{array}$ & Fever, malaria & 0.93 \\
\hline Celosia argentea $\mathrm{L}$ & Amaranthaceae & Bungo bayom & Herb & Cultivated & Leaves & Decoction & Hypertension & 0.78 \\
\hline Centella asiatica (L.) Urb. & Apiaceae & Pegago & Herb & Cultivated & Whole plants & Decoction & Cough & 0.58 \\
\hline $\begin{array}{l}\text { Chromolaena odorata (L.) } \\
\text { R.M.King \& H.Rob. }\end{array}$ & Asteraceae & Tutuba & Shrub & Wild & Leaves & $\begin{array}{l}\text { Decoction, pounded, } \\
\text { smeared }\end{array}$ & Gastric ulcer, wounds & 0.37 \\
\hline Citrus maxima (Burm.) Merr. & Rutaceae & Jeruk bali & Tree & Cultivated & Fruit & Raw consumption & Sprue & 0.82 \\
\hline $\begin{array}{l}\text { Clerodendrum chinense (Osbeck) } \\
\text { Mabb. }\end{array}$ & Lamiaceae & Bungo balai & Shrub & Wild & Flower & Decoction & Sore throat, sprue & 0.63 \\
\hline Clerodendrum indicum (L.) Kuntze & Lamiaceae & Rumput pikuben & Shrub & Wild & Leaves, flower & Dec & Gas & 0.27 \\
\hline Clerodendrum thomsoniae Balf.f. & Lamiaceae & Kantin & Shrub & Wild & Flower & Decoction & Irritant contact dermatitis due to plants & 0.28 \\
\hline $\begin{array}{l}\text { Clinacanthus nutans (Burm.f.) } \\
\text { Lindau }\end{array}$ & Acanthaceae & Sogi ijau & Herb & Cultivated & Leaves & Decoction & Sore throat, sprue & 0.27 \\
\hline Cocos nucifera $\mathrm{L}$. & Arecaceae & Karambie & Palm & Cultivated & Fruit & Decc & oea, digestive problems, constipation & 0.77 \\
\hline Coleus amboinicus Lour. & Lamiaceae & Nilam babi & Herb & Wild & Leaves & Decoction & Hepatitis & 0.72 \\
\hline Colubrina asiatica (L.) Brongn. & Rhamnaceae & Pilaut & Shrub & Cultivated & Leaves & Decoction & Fever, sore throat & 0.53 \\
\hline Combretum indicum (L.) DeFilipps & Combretaceae & Pocah pingen & Climber & Wild & Leaves, flower & Decoction & Anthelmintic & 0.42 \\
\hline Cordyline fruticosa (L.) A.Chev. & Asparagaceae & Junjuang & Shrub & Cultivated & Whole plants & Smeared & Bruise, wounds & 0.32 \\
\hline
\end{tabular}




\begin{tabular}{|c|c|c|c|c|c|c|c|c|}
\hline Crinum asiaticum $\mathrm{L}$. & Amaryllidaceae & Babakuong & Herb & Cultivated & Leaves, fruit & Smeared. decoction & Wounds, bruise, rheumatism & 0.35 \\
\hline Cucumis melo $\mathrm{L}$. & Cucurbitaceae & Melon & Herb & Cultivated & Fruit & Raw consumption & Hypertension & 0.30 \\
\hline Cyclea barbata Miers & Menispermaceae & Cincau & Shrub & Cultivated & Leaves & Decoction & Sore throat, sprue & 0.55 \\
\hline Cymbopogon citratus (DC.) Stapf & Poaceae & Sereh & Grass & Cultivated & Whole plants & Decoction & Sore throat, sprue, hypertension & 0.72 \\
\hline Cyperus rotundus L. & Cyperaceae & Umpuik lading & Grass & Wild & Leaves, flower & Decoction, squeezed & Cough & 0.45 \\
\hline $\begin{array}{l}\text { Decalobanthus mammosus (Lour.) } \\
\text { A.R.Simões \& Staples }\end{array}$ & Convolvulaceae & Bidaro upas & Climber & Wild & Tuber & Pounded, decoction & Cough, bruise & 0.60 \\
\hline Dioscorea alata $\mathrm{L}$. & Dioscoreaceae & Gaduong angina & Climber & Wild & Tuber & Pounded, decoction & Sore throat, sprue & 0.48 \\
\hline Dioscorea hispida Dennust. & Dioscoreaceae & Gaduong tanah & Climber & Wild & Tuber & Pounded, decoction & Sore throat, sprue & 0.60 \\
\hline Durio zibethinus L. & Malvaceae & Drien & Tree & Cultivated & Fruit & Raw consumption & Anemia & 0.27 \\
\hline $\begin{array}{l}\text { Erythrina subumbrans (Hassk.) } \\
\text { Merr. }\end{array}$ & Fabaceae & Reudeup & Tree & Wild & $\begin{array}{l}\text { Leaves, flower, } \\
\text { fruit }\end{array}$ & Decoction & Sore throat, hepatitis & 0.42 \\
\hline Erythrina variegata $\mathrm{L}$. & Fabaceae & Dodok & Tree & Wild & $\begin{array}{l}\text { Leaves, flower, } \\
\text { fruit }\end{array}$ & Decoction & Jaundice & 0.38 \\
\hline Euphorbia hirta L. & Euphorbiaceae & Didieh & Herb & Wild & Whole plants & Dropped & Eye inflammations & 0.47 \\
\hline Gomphrena globosa $\mathrm{L}$. & Amaranthaceae & Bungo kenop & Herb & Cultivated & Flower & Decoction & Fever, cough, dysentery & 0.43 \\
\hline Gynura japonica (Thunb.) Juel & Asteraceae & Daun inggu & Herb & Wild & Leaves & Decoction & Influenza, malaria & 0.27 \\
\hline $\begin{array}{l}\text { Hellenia speciosa (J.Koenig) } \\
\text { S.R.Dutta }\end{array}$ & Costaceae & Sitawa & Shrub & Wild & Leaves & Decoction & Gastric ulcer & 0.22 \\
\hline Hibiscus rosa-sinensis $\mathrm{L}$. & Malvaceae & Kembang sepatu & Shrub & Cultivated & Flower & Decoction & Gastric ulcer & 0.32 \\
\hline Hippobroma longiflora (L.) G.Don & Campanulaceae & Katarak & Herb & Cultivated & Leaves, flower & Dropped & Eye inflammations & 0.27 \\
\hline Illicium verum Hook.f. & Schisandraceae & Bunga lawang & Tree & Cultivated & Fruit & Decoction & Flatulence, fever & 0.77 \\
\hline Imperata cylindrica (L.) P.Beauv. & Poaceae & Rumput Padang & Grass & Wild & Whole plants & Decoction & Fever, sore throat, sprue & 0.87 \\
\hline Jasminum sambac (L.) Aiton & Oleaceae & Pandak kaki & Tree & Cultivated & Leaves, flower & Dropped & Eye inflammations & 0.65 \\
\hline Jatropha podagrica Hook. & Euphorbiaceae & Jiyak bali & Tree & Cultivated & Sap & Dropped & Wounds, toothache & 0.73 \\
\hline Justicia gendarussa Burm.f & Acanthaceae & Sogi itom & Herb & Cultivated & Leaves & Decoction & Sore throat & 0.53 \\
\hline Kaempferia galanga $\mathrm{L}$. & Zingiberaceae & Kencur & Herb & Cultivated & Tuber & Decoction & Cough, fever & 0.57 \\
\hline Kalanchoe pinnata (Lam.) Pers. & Crassulaceae & Si dingin & Herb & Cultivated & Leaves & Affixed to the forehead & Fever & 0.38 \\
\hline Lansium domesticum Corrêa & Meliaceae & Langsat & Tree & Cultivated & Seed & Raw consumption & Malaria & 0.47 \\
\hline Lawsonia inermis L. & Lythraceae & Inai & Shrub & Cultivated & Leaves & Decoction & Gastric ulcer & 0.35 \\
\hline Lophatherum gracile Brongn. & Poaceae & Mimilang & Grass & Wild & Whole plants & Decoction & Sore throat & 0.27 \\
\hline Luffa acutangula (L.) Roxb. & Cucurbitaceae & Patulo & Climber & Cultivated & Fruit & Decoction & Sore throat, anthelmintic & 0.27 \\
\hline Luffa aegyptiaca Mill. & Cucurbitaceae & Pio & Climber & Cultivated & Fruit & Decoction & Fever & 0.23 \\
\hline Magnolia $\times$ alba $(\mathrm{DC}$.$) Figlar$ & Magnoliaceae & Cempaka putih & Tree & Cultivated & Flower & Dropped & Eye inflammations & 0.37 \\
\hline $\begin{array}{l}\text { Magnolia champaca (L.) Baill. ex } \\
\text { Pierre }\end{array}$ & Magnoliaceae & Nangoe & Tree & Cultivated & Flower & Decoction & Gastric ulcer & 0.45 \\
\hline Manilkara zapota (L.) P.Royen & Sapotaceae & Sawoh & Tree & Wild & Leaves & Decoction & Hypertension, diarrhea & 0.28 \\
\hline Melastoma malabathricum L. & Melastomataceae & Kaduduok & Shrub & Wild & Leaves & Smeared & Wounds & 0.73 \\
\hline Mirabilis jalapa $\mathrm{L}$ & Nyctaginaceae & $\begin{array}{l}\text { Bungo } \\
\text { kombangsubuh }\end{array}$ & Herb & Cultivated & Whole plants & Decoction & Gastric ulcer, pimple & 0.85 \\
\hline Morinda citrifolia $\mathrm{L}$. & Rubiaceae & Mengkudu & Tree & Cultivated & Fruit & $\begin{array}{l}\text { Raw consumption, } \\
\text { decoction }\end{array}$ & $\begin{array}{l}\text { Stroke, Influenza, headache, diabetes, } \\
\text { hypertension, rheumatism }\end{array}$ & 0.95 \\
\hline Murraya koenigii (L.) Spreng. & Lamiaceae & Salam koja & Shrub & Wild & Leaves & Decoction & Diarrhoea, diabetes, sore throat & 0.73 \\
\hline Myristica fragrans Houtt. & Myristicaceae & Buah palo & Tree & Cultivated & Seed & Pounded, smeared & Sprain & 0.77 \\
\hline
\end{tabular}




\begin{tabular}{|c|c|c|c|c|c|c|c|c|}
\hline Nicandra physalodes (L.) Gaertn. & Solanaceae & Lolotuok & Herb & Wild & Fruit & Decoction & Hypertension & 0.38 \\
\hline Nicotiana tabacum $\mathrm{L}$. & Solanaceae & Tembakau & Herb & Cultivated & Leaves & Decoction & Jaundice & 0.30 \\
\hline Nypa fruticans Wurmb & Arecaceae & Nipah & Palm & Wild & Leaves & Decoction & Jaundice & 0.37 \\
\hline Ocimum tenuiflorum $\mathrm{L}$. & Lamiaceae & Kemangi & Shrub & Cultivated & Whole plants & Raw consumption & Fever, sore throat & 0.88 \\
\hline Oldenlandia corymbosa $\mathrm{L}$. & Rubiaceae & Sititom & Herb & Wild & Leaves & Decoction & Hepatitis & 0.55 \\
\hline Orthosiphon aristatus (Blume) Miq. & Fabaceae & Kumis kucing & Herb & Cultivated & Leaves, flower & Decoction & Diabetes, hypertension, bladder stone & 0.47 \\
\hline Pachyrhizus erosus (L.) Urb. & Fabaceae & Bengkuang & Climber & Cultivated & Tuber & Raw consumption & Influenza, sore throat & 0.60 \\
\hline Paederia foetida $\mathrm{L}$. & Rubiaceae & Daun kentut & Climber & Wild & Whole plants & Decoction & Flatulence & 0.38 \\
\hline $\begin{array}{l}\text { Pandanus amaryllifolius Roxb. ex } \\
\text { Lindl. }\end{array}$ & Pandanaceae & Panden musang & Shrub & Cultivated & Leaves & Decoction & Fever, insomnia, hypertension & 0.65 \\
\hline Persea americana Mill. & Lauraceae & Pukat & Tree & Cultivated & Fruit & Raw consumption & Eye inflammations & 0.37 \\
\hline Phyllanthus acidus (L.) Skeels & Phyllanthaceae & Ceremai & Tree & Cultivated & Fruit & Raw consumption & Sprue & 0.32 \\
\hline Phyllanthus niruri L. & Phyllanthaceae & Dukong anak & Herb & Wild & Whole plants & Decoction & Gastric ulcer, diabetes & 0.45 \\
\hline Piper betle L. & Piperaceae & Sirih & Climber & Cultivated & Leaves & $\begin{array}{l}\text { Decoction, pounded, } \\
\text { smeared }\end{array}$ & Fever, sore throat, wounds & 0.98 \\
\hline Plantago major $\mathrm{L}$. & Plantaginacae & Tutup bumi & Herb & Cultivated & Whole plants & Decoction & Cough & 0.78 \\
\hline Plectranthus purpuratus Harv. & Lamiaceae & Paladang & Herb & Cultivated & Whole plants & Decoction & Gastric ulcer, hemorrhoids, hepatitis & 0.63 \\
\hline Pluchea indica (L.) Lees. & Asteraceae & Capa & Herb & Wild & Whole plants & Decoction & Flatulence, hepatitis & 0.37 \\
\hline Psidium guajava $\mathrm{L}$. & Myrtaceae & Asomsogi & Tree & Cultivated & Fruit, leaves & $\begin{array}{l}\text { Raw consumption, } \\
\text { decoction }\end{array}$ & Dengue, diarrhea & 0.98 \\
\hline Punica granatum $\mathrm{L}$. & Punicaceae & Delima & Tree & Cultivated & Fruit & Eaten raw & Stroke, sore throat & 0.60 \\
\hline Ricinus communis $\mathrm{L}$. & Euphorbiaceae & Jiyak & Tree & Cultivated & Sap & Dropped, decoction & Wounds, hernia & 0.73 \\
\hline Selaginella doederleinii Hieron & Selaginellaceae & Paku ayom & Herb & Wild & Whole plants & Decoction & Hepatitis & 0.47 \\
\hline Senna alexandrina Mill. & Fabaceae & Galinggang & Shrub & Wild & Leaves & Decoction & Scabies, & 0.43 \\
\hline Solanum lasiocarpum Dunal & Solanaceae & Terong & Shrub & Cultivated & Fruit & Eaten as vegetable & Diabetes & 0.58 \\
\hline Solanum nigrum $\mathrm{L}$. & Solanaceae & Rimbang & Shrub & Cultivated & Fruit, leaves & Decoction, dropped & Eye inflammations & 0.63 \\
\hline Spondias dulcis Parkinson & Anacardiaceae & Kadunduong & Tree & Cultivated & Fruit & $\begin{array}{l}\text { Raw consumption, } \\
\text { decoction, dropped }\end{array}$ & Anemia, eye inflammations & 0.52 \\
\hline Syzygium cumini (L.) Skeels & Myrtaceae & Jambu kleng & Tree & Cultivated & Leaves, Fruit & $\begin{array}{l}\text { Decoction, Raw } \\
\text { consumption }\end{array}$ & Diabetes, constipation & 0.97 \\
\hline Tamarindus indica $\mathrm{L}$. & Fabaceae & Asam jawa & Tree & Cultivated & Fruit & Raw consumption & Sore throat, sprue & 0.95 \\
\hline Trema orientale (L.) Blume & Cannabaceae & Sitopuong & Tree & Wild & Leaves & Decoction & Cough, asthma, sore throat, fever & 0.75 \\
\hline Uncaria gambir (W.Hunter) Roxb. & Rubiaceae & Sibubuik & Climber & Wild & Leaves & Decoction & Flatulence & 0.73 \\
\hline
\end{tabular}


The second-largest proportion of plant parts used by the Aneuk Jamee tribe as traditional medicine is fruit Averrhoa bilimbi fruit is used by the Aneuk Jamee tribe as a hypertension treatment. Susanti et al. (2017) reported that the extract of $A$. bilimbi fruit could reduce blood glucose levels and can be used as a treatment for diabetes. A. bilimbi is known to contain flavonoids and saponins that act as antidiabetic agents (Kumar et al. 2013). The natural diuretic activity of $A$. bilimbi plays a significant role in combating hypertension (Andriyanto et al. 2011). Fruit of Morinda citrifolia is used for stroke, influenza, headache, diabetes, hypertension, and rheumatism, while Solanum lasiocarpum fruit is used for diabetes.

\section{Mode of preparation}

The major mode of preparation by the Aneuk Jamee tribe was found to be decoction $(60 \%)$, followed by raw consumption $(15 \%)$, smeared $(10 \%)$, and pounded $(7 \%)$ (Figure 3).

In order to treat diseases, the Aneuk Jamee tribe applied both internal and external administration routes. Most plant species have been used alone in the treatment of diseases, while several plants are combined. For example, Oldenlandia corymbosa was used alone in the treatment of hepatitis, while Centella asiatica was administered orally for cough treatment in combination with honey. In Indian folk medicine, the $C$. asiatica are useful for the treatment of asthma, skin disorders, gastric ulcer and body aches, gastric catarrh, kidney troubles, leprosy, stomach disorders, cure dysentery, and improve memory power (Jamil et al. 2007), while in Nepal, the leaf juice mixed with palm leaves used for cooling to body and stomach (Mahato and Chaudhary 2003).

\section{Use value}

The use-value (UV) of plants has been calculated to quantify the importance of a specific plant on the basis of how often it is cited by a specific number of people. UV scores ranged from 0.13 to 0.97 , with Hellenia speciosa having lower UV (0.22) and Psidium guajava and Piper betle having the highest UV (0.97 each). P. guajava and $P$. betle are widely used by the Aneuk Jamee tribe to treat various diseases. P. guajava decoction has been used by the Aneuk Jamee tribe to treat various diseases such as dengue and diarrhoea. P. guajava is also the most widely used medicinal plant in many countries, such as Mexico, Africa, Asia and Central America (Naseer et al. 2018). In Andhra Pradesh, India, leaves of guava have been documented for use in mouth gastric ulcer (Lingaiah and Rao 2013), while in North Sikkim, India, raw young leaves and tender shoots of guava have been used for toothache and mouth gastric ulcer (Pradhan and Badola 2008). The decoction of leaves of $P$. betle is used to treat fever and sore throat, while leaves of $P$. betle were squeezed and placed on the wounds. $P$. betle leaf extracts contain bioactive compounds, such as sterol (Pradhan et al. 2013), which are responsible for the antibacterial activity and are suitable for wounds. Essential oil from the leaves of this plant has been used for antiseptic treatment (Amalia et al. 2008). In the traditional Indian system, $P$. betle leaves are used as digestive and pancreatic lipase stimulating activity (Mula et al. 2008).

\section{Informant consensus factor}

Diseases reported by respondents have been classified according to the International Classification of Diseases 10 ver. 2019 (https: //icd.who.int). Out of these categories, ICF values were determined and shown in Table 3.

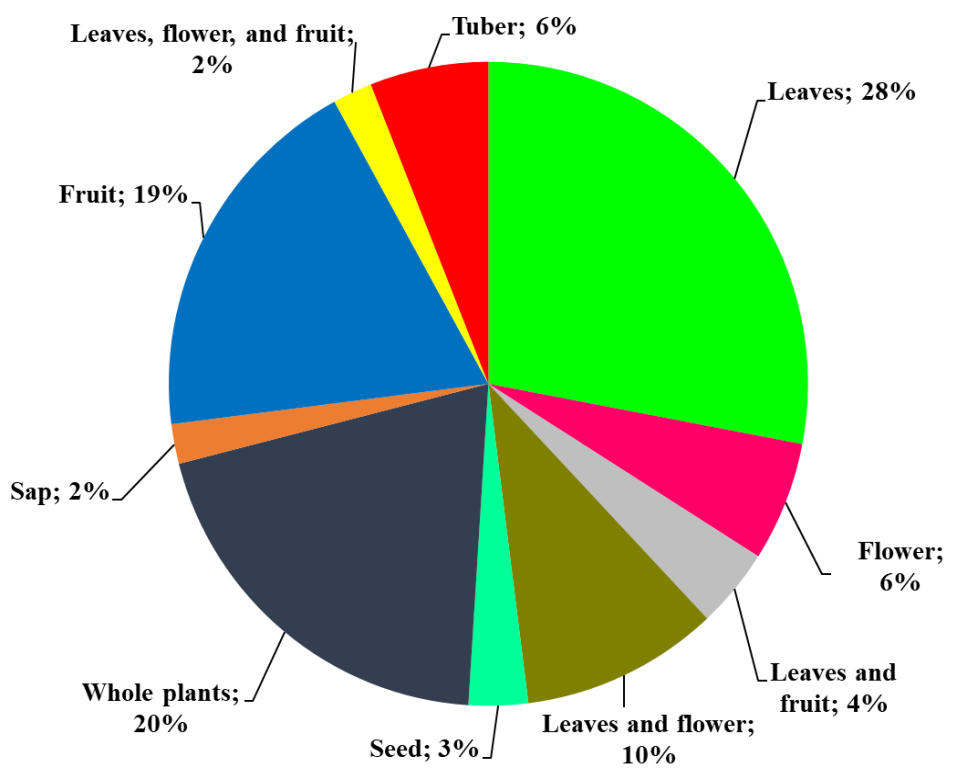

Figure 2. Plant part used by the Aneuk Jamee tribe in Kota Bahagia Sub-district, South Aceh, Indonesia 


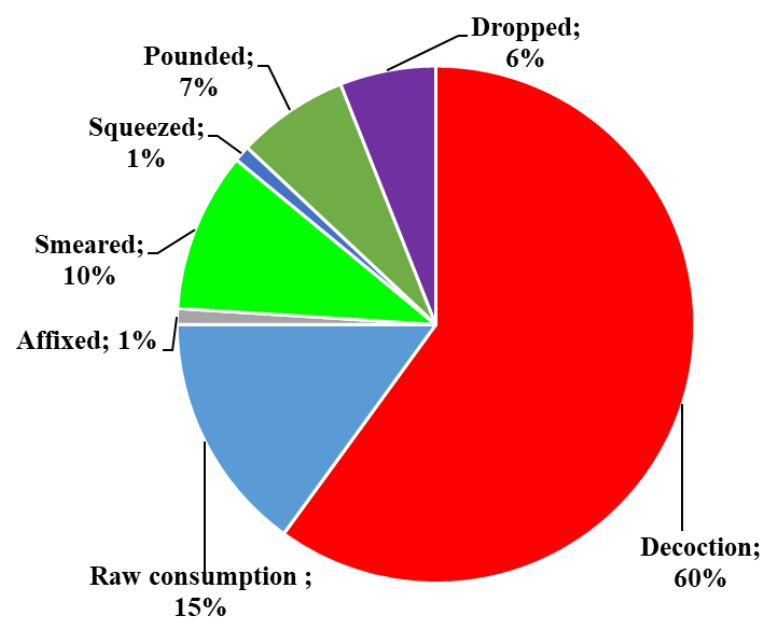

Figure 3. Mode of preparation of medicinal plants using by Aneuk Jamee tribe in Kota Bahagia Sub-district, South Aceh, Indonesia

Table 3. Categories of disease in the study area and the informant consensus factor (ICF)

\begin{tabular}{|c|c|c|c|c|}
\hline Classification of diseases & Specified disease name & $\begin{array}{c}\text { Number } \\
\text { use-report }\end{array}$ & $\begin{array}{c}\text { Number } \\
\text { species }\end{array}$ & $\begin{array}{l}\text { ICF } \\
\text { value }\end{array}$ \\
\hline Certain infectious and parasitic diseases (CID) & $\begin{array}{l}\text { Dysentery, diarrhea, ringworm, scabies, } \\
\text { tuberculosis, malaria, dengue, anthelmintic }\end{array}$ & 386 & 13 & 0.973 \\
\hline Diseases of liver (LD) & Hepatitis & 152 & 7 & 0.960 \\
\hline Diseases of the circulatory system (CSD) & Hemorrhoid, hypertension, stroke & 56 & 3 & 0.964 \\
\hline Diseases of the digestive system (DSD) & $\begin{array}{l}\text { Constipation, hernia, gastric ulcer, } \\
\text { stomachache, toothache, haematemesis }\end{array}$ & 503 & 21 & 0.960 \\
\hline Diseases of the eye and adnexa (EAD) & Eye inflammation & 441 & 18 & 0.961 \\
\hline Diseases of the genitourinary system (GD) & $\begin{array}{l}\text { Dysmenorrhea, leukorrhea, bladder stone, } \\
\text { menstrual pain, vaginal discharge }\end{array}$ & 56 & 3 & 0.964 \\
\hline $\begin{array}{l}\text { Diseases of the musculoskeletal system and } \\
\text { connective tissue (MCD) }\end{array}$ & Rheumatism & 38 & 3 & 0.946 \\
\hline Diseases of the respiratory system (RSD) & Asthma, influenza, sore throat, sprue & 631 & 25 & 0.962 \\
\hline $\begin{array}{l}\text { Diseases of the skin and subcutaneous tissue } \\
\text { (DS) }\end{array}$ & $\begin{array}{l}\text { Itch, swelling, irritant contact dermatitis } \\
\text { due to plants }\end{array}$ & 61 & 4 & 0.950 \\
\hline $\begin{array}{l}\text { Diseases of the blood and blood-forming } \\
\text { organs and certain disorders involving the } \\
\text { immune mechanism (DBF) }\end{array}$ & Anemia & 56 & 2 & 0.982 \\
\hline $\begin{array}{l}\text { Endocrine, nutritional and metabolic diseases } \\
\text { (ENM) }\end{array}$ & Diabetes & 145 & 8 & 0.951 \\
\hline $\begin{array}{l}\text { Injury, poisoning, and certain other } \\
\text { consequences of external causes (IPD) }\end{array}$ & Wound, bruise, sprain & 259 & 10 & 0.965 \\
\hline Mental and behavioral disorders (MBD) & Insomnia & 34 & 3 & 0.939 \\
\hline $\begin{array}{l}\text { Symptoms and signs involving the circulatory } \\
\text { and respiratory systems (DCR) }\end{array}$ & Cough, nosebleed & 271 & 10 & 0.967 \\
\hline $\begin{array}{l}\text { Symptoms and signs involving the skin and } \\
\text { subcutaneous tissue (SCT) }\end{array}$ & Skin burn & 100 & 4 & 0.970 \\
\hline $\begin{array}{l}\text { Symptoms, signs, and abnormal clinical and } \\
\text { laboratory (SSA) }\end{array}$ & $\begin{array}{l}\text { Fever, cold, flatulence, pimple, jaundice, } \\
\text { headache }\end{array}$ & 584 & 24 & 0.961 \\
\hline
\end{tabular}

A total of 47 diseases in 16 categories were documented in the study area. The most common use-report categories are diseases of the respiratory system (631 use-report, 25 species), followed by symptoms, signs and abnormal clinical and laboratory (584 use-reports, 24 species), diseases of the digestive system (503 use-reports, 21 species), diseases of the eye and adnexa (441 use-reports, 18 species), and certain infectious and parasitic diseases (386 use-reports, 13 species). The ICF values ranged from 0.939 to 0.982 . The highest ICF value $(0.982)$ is for diseases of the blood and blood-forming organs and certain disorders involving the immune mechanism (anemia), while the lowest is for mental and behavioral disorders (0.939). Sandjaja et al. (2013) reported that the prevalence of anemia in rural areas is higher than in urban areas. This is affected by the consumption pattern of rural communities, which is still dominated by vegetables as a source of iron (non-heme iron), resulting in low use and absorption of iron. The respondents cited two species of plants for the treatment of anemia, i.e., Spondias dulcis and 
Durio zibethinus. During the discussion, the respondents stated that the Aneuk Jamee tribe often consumes the fruit of $D$. zibethinus and the young fruits of $S$. dulcis which are believed to prevent anemia. D. zibethinus fruit is reported to be rich in nutrients including antioxidants that are important in the prevention of anemia (Amir and Saleh 2014), while $S$. dulcis fruits are rich in bioactive compounds and used in traditional medicines in Sri Lanka, India, Vietnam, and Malaysia to treat anemia, regulate blood glucose levels, and digestive problems (Jayarathna et al. 2020).

Traditional knowledge of medicinal plants in the Aneuk Jamee tribe has been passed down from generation to generation. This traditional knowledge, however, is not well documented. Transfer of knowledge is still being carried out orally. However, during discussions with the respondents, it was noted that many children were interested in learning medicinal plants with healers or elders in their village. This traditional knowledge needs to be protected by involving the Indonesian Government through the Education Office with the integration of traditional knowledge into the basic education curriculum. This practice could be an effort to preserve traditional knowledge, natural resources, and biodiversity. Ramadoss and Moli (2011) reported that biodiversity education programs could increase student's knowledge, motivation, and expertise to conserve and protect local natural resources and biodiversity in India.

\section{ACKNOWLEDGEMENTS}

We are grateful to all the respondents in the study area for their kind friendliness and knowledge sharing. We are also thankful that this study has been supported by Samudra University, Indonesia.

\section{REFERENCES}

Abbink J. 1995. Medicinal and ritual plants of the Ethiopian Southwest: An account of recent research. Indigenous Knowledge and Development Monitor 3 (2): 6-8

Amalia H, Sitompul R, Hutauruk J, Andrianjah, Mun'im A. 2008. Effectiveness of Piper betle leaf infusion as a palpebral skin antiseptic. Universa Medicina 28 (2): 83-91

Amir F, Saleh C. 2014. Antioxidant activity test of ethanol extract from Durio zibethinus Murr seeds by DPPH method. Jurnal Kimia Mulawarman 11 (2): 84-87

Andriyanto, Kusumorini N, Yuskha F. 2011. The potency of ethanolic extract of Bilimbi (Averrhoa bilimbi L.) fruits as a natural diuretic. Jurnal Ilmu Kefarmasian Indonesia 9 (2): 78-84

Bekalo TH, Woodmatas SD, Woldemariam ZA. 2009. An ethnobotanical study of medicinal plants used by local people in the lowlands of Konta Special Woreda, southern nations, nationalities and peoples regional state, Ethiopia. J Ethnobiol Ethnomed 5: 26

Calzada F, Bautista E. 2020. Plants used for the treatment of diarrhoea from Mexican flora with amoebicidal and giadicidal activity, and their phytochemical constituents. J. Ethnopharmacology 253: 112676

Colalto C. 2018. What phytotherapy needs: Evidencebased guidelines for better clinical practice. Phytother. Res. 32 (3): 413-425

Cornara L, La Rocca A, Terrizzano L, Dente F, Mariotti MG. 2014. Ethnobotanical and phytomedical knowledge in the North-Western Ligurian Alps. J Ethnopharmacol 155 (1): 463-84
Dar RA, Shahnawaz M, Qazi PH. 2017. General overview of medicinal plants: A review. The J Phytopharmacology 6 (6): 349-351

Elfahmi, Woerdenbag H, Kayser O. 2014. Jamu: Indonesian traditional herbal medicine towards rational phytopharmacological use. J Herbal Med 4 (2): 51-73

Elfrida, Mubarak A, Suwardi AB. 2020. The fruit plant species diversity in the home gardens and their contribution to the livelihood of communities in rural area. Biodiversitas 21 (8): 3670-3675.

Ezekwesili JO, Nkemdilim UU, Okeke UU. 2010. Mechanism of antidiarrhoeal effect of ethanolic extract of Psidium guajava leaves. Biokemistri 22 (2): 85-90.

Ghorbani A. 2005. Studies in pharmaceutical ethnobotany in the region of Turkmen Sahra, North of Iran (part 1): general results. J Ethnopharmacol 102: 58-68.

Gowramma B, Kyagavi G, Karibasamma H, Ramanjinaiah KM. 2020. Documentation of major medicinal plants in Sandure of Karnataka, India. Med Aromat Plants 9: 348

Ismail A, Ahmad WANW. 2019. Syzygium polyanthum (Wight) Walp: A Potential Phytomed Pharm J 11 (2): 429-438.

Jamil SS, Nizami Q, Salam M. 2007. Centella asiatica (Linn.) Urban: areview. Indian J Nat Prod Resour 6 (2): 158-170.

Jayarathna PLI, Jayawardena JAEC, Vanniarachchy MPG. 2020. Identification of physical, chemical properties and flavor profile of Spondias dulcis in three maturity stages. Intl Res J Adv Eng Sci 5 (1): 208-211.

Kumar KA, Gousia SK, Anupama M, Latha JNL. 2013. A review on phytochemical constituents and biological assays of Averrhoa bilimbi. Intl J Pharm Pharmaceut Sci Res 3 (4): 136-139.

Kumar S, Singh A, Singh B, Maurya R, Kumar B. 2018. Structural characterization and quantitative determination of bioactive compounds in ethanolic extraction of Boerha avia diffusa by liquid chromatography-tandem mass spectrometry. J Sep Sci Plus 1 (9): 588-596.

Lingaiah M, Rao PN. 2013. An ethnobotanical survey of medicinal plants used by traditional healers of Adilabad district, Andhra Pradesh, India. Biolife 1: 17-23

Liu Y, Dao Z, Yang C, Liu Y, Long C. 2009. Medicinal plants used by Tibetans in Shangri-la, Yunnan, China. J Ethnobiol Ethnomed 5: 15. DOI: $10.1186 / 1746-4269-5-15$.

Mahato RB, Chaudhary RP.2003. Ethnomedicinal study and antibacterial activities of selected plants of Palpa District, Nepal. Sci World 2: 3845

Maulidiah, Winandari OP, Saputri DA. 2020. Utilization of plant organs as traditionally processed medicines in Kebun Tebu Subdistrict, West Lampung District. Jurnal Ilmu Kedokteran dan Kesehatan 7 (2): 443 447

Melalatoa MJ. 1995. Encyclopedia of Ethnic Groups in Indonesia. Ministry of Education and Culture of the Republic of Indonesia, Jakarta. [Indonesian]

Mula S, Banerjee D, Patro BS, Barik A, Bandopadhayay SK, Chattopadhyay S. 2008. Inhibitory property of the Piper betle phenolics against photosensitization induced biological damages. Bioorg Med Chem 16: 2932-2938.

Naseer S, Hussain S, Naeem N, Pervaiz M, Rahman M. 2018. The phytochemistry and medicinal value of Psidium guajava (guava). Clin Phytosci 4: 32. DOI: 10.1186/s40816-018-0093-8.

Navia ZI, Audira D, Afifah N, Turnip K, Nuraini, Suwardi AB. 2020 b. Ethnobotanical investigation of spice and condiment plants used by the Taming tribe in Aceh, Indonesia. Biodiversitas 21 (10): 4467 4473 .

Navia ZI, Chikmawati T. 2015. Durio tanjungpurensis (Malvaceae), a new species and its one new variety from West Kalimantan, Indonesia. Bangladesh J Bot 44 (3): 429-436.

Navia ZI, Suwardi AB, Harmawan T, Syamsuardi, Mukhtar E. 2020a. The diversity and contribution of indigenous edible fruit plants to the rural community in the Gayo Highlands, Indonesia. J Agric Rural Dev Trop Subtrop 121 (1): 89-98.

Navia ZI, Suwardi AB, Saputri A. 2019. Characterization of local fruits in the Leuser Ecosystem of Aceh Taming District, Aceh. Buletin Plasma Nutfah 25 (2): 133-142.

Ngbolua KN, Lufuluabo LG, Moke LE, Bongo GN, Liyongo CI, Ashande CM, Sapo BS, Zoawe BG, Mpiana PT. 2018.A review on the Phytochemistryand Pharmacology of Psidium guajava L. (Myrtaceae) and Future direction. Discovery Phytomedicine 5 (2): 7-13.

Ngbolua KN, Mihigo SO, Mpiana PT, Inkoto CL, Masengo CA, Tshibangu DST, Gbolo BZ, Baholy R,Fatiany PR. 2016. Ethno- 
pharmacological survey and ecological studies of some plants used in traditional medicine in Kinshasa city (Democratic Republic of the Congo). Trop Plant Res 3 (2) : 413-427.

Nurlinda, Payung I, Juana P, Suwardi AB. 2018. Anti-microfilarial activity of rhizome extract of Curcuma aerugenosa Roxb. (Zingiberaceae). J Chem Pharmaceut Res 10 (8): 33-36.

Ortiz AC, Musarella CM, Gomes CJP, Canas RQ, Fuentes JCP, Cano E. 2020. Phytosociological study, diversity and conservation status of the Cloud Forest in the Dominican Republic. Plants 9: 741. DOI: $10.3390 /$ plants9060741.

Polat R, Cakilcioglu U, Kaltaliołlu K, Ulusan MD, Türkmen Z. 2015. An ethnobotanical study on medicinal plants in Espiye and its surrounding (Giresun-Turkey). J Ethnopharmacol 163: 1-11.

Pradhan BK, Badola HK. 2008. Ethnomedicinal plant use by Lepcha tribe of Dzongu valley, bordering Khangchendzonga Biosphere Reserve, in North Sikkim, India. J Ethnobiol Ethnomed 4: 22. DOI: 10.1186/1746-4269-4-22.

Pradhan D, Suri KA, Pradhan DK, Biswasroy P. 2013. Golden Heart of the Nature: Piper betle L. J Pharmacogn Phytochem 1 (6): 147-167.

Ramadoss A, Moli GP. 2011. Biodiversity Conservation through Environmental Education for Sustainable Development: A Case Study from Puducherry, India. Intl Electr J Environ Educat 1 (2): 97 111.

Ramli NFN, Erwandi N. 2019. Comparative analysis between Jamee (Aceh) and Minangkabau (Bukit Tinggi) language. Linguistik Indonesia 37 (1): 81-95. [Indonesian]

Sandjaja S, Budiman B, Harahap H, Ernawati F, Soekatri M, Widodo Y, Sumedi E, Rustan E, Sofia G, Syaries SN, Khouw I. 2013. Food consumption and nutritional and biochemical status of 0.5-12-yearold Indonesian children: the SEANUTS study. Br J Nutr 110: S11S20.

Sari ID, Yuniar Y, Siahaan S, Riswati, Syaripuddin M. 2015. Community tradition in planting and using medicinal plant in surround home yard. Jurnal Kefarmasian Indonesia 5 (2): 123-132

Saynes-Váquez A, Vibrans H, Vergara-Silva F, Caballero J. 2016 Intracultural differences in local botanical knowledge and knowledge loss among the Mexican Isthmus Zapotecs. PLoS ONE 11 (3): e0151693. DOI: 10.1371/journal.pone.0151693.

Shakeera BM, Sujatha K, Sridharan G, Manikandan R.2013. Antihyperglycemic and antihyperlipidemic potentials of Psidium guajava in alloxan-induced diabetic rats. Asian J Pharmaceut Clin Res 6 (2): 88-89.

Silalahi M, Supriatna J, Walujo EB, Nisyawati. 2015. Local knowledge of medicinal plants in sub-ethnic Batak Simalungun of North Sumatra, Indonesia. Biodiversitas 16 (1): 44-54.
Sousa RS, Hanazaki N, Lopes JB, de Barros RFM. 2012. Are gender and age important in understanding the distribution of local botanical knowledge in fishing communities of the Parnaíba Delta Environmental Protection Area? Ethnobot Res Appl 10: 551-559.

Susanti EY, Candra A, Nissa C. 2017. Effect of Belimbing Wuluh (Averrhoa bilimbi L.) extracts on fasting blood glucose levels in adult women. J Nutrition Health 5 (2): 102-115.

Sutrisno IH, Akob B, Navia ZI, Nuraini, Suwardi AB, 2020. Documentation of ritual plants used among the Aceh tribe in Peureulak, East Aceh District, Indonesia. Biodiversitas 21 (11): 49904998

Suwardi AB, Indriaty, Navia ZI. 2018. Nutritional evaluation of some wild edible tuberous plants as an alternative foods. Innovare J Food Sci 6 (2): 9-12.

Suwardi AB, Navia ZI, Harmawan T, Syamsuardi, Mukhtar E. 2019. The diversity of wild edible fruit plants and traditional knowledge in West Aceh region, Indonesia. J Med Plants Stud 7 (4): 285-290.

Suwardi AB, Navia ZI, Harmawan T, Syamsuardi, Mukhtar E. 2020a. Wild edible fruits generate substantial income for local people of the Gunung Leuser National Park, Aceh Tamiang Region. Ethnobot Res Appl 20: 1-13.

Suwardi AB, Navia ZI, Harmawan T, Syamsuardi, Mukhtar E. 2020b. Ethnobotany, nutritional composition and sensory evaluation of Garcinia from Aceh, Indonesia. Mater Sci Eng 725 (1): 012064 . DOI: 10.1088/1757-899X/725/1/012064.

Suwardi AB, Navia ZI, Harmawan T, Syamsuardi, Mukhtar E. 2020c. Ethnobotany and conservation of indigenous edible fruit plants in South Aceh, Indonesia. Biodiversitas 21 (5): 1850-1860.

Tantengco OAG, Condes MLC, Estadilla HHT, Ragragio EM. 2018. Ethnobotanical Survey of Medicinal Plants used by Ayta Communities in Dinalupihan, Bataan, Philippines. Pharmacogn J 10 (5): 859-870.

The Central Bureau of Statistics of South Aceh District. 2020. South Aceh district in figure 2019. The Central Bureau of Statistics of South Aceh district, Indonesia.

Tsioutsiou EE, Giordani P, Hanlidou E, Biagi M, de Feo V, Cornara L. 2019. Ethnobotanical Study of Medicinal Plants Used in Central Macedonia, Greece. Evid Based Compl Alternat Med 2019: 4513792. DOI: $10.1155 / 2019 / 4513792$.

Yeung AWK, Heinrich M, Kijjoa A, Tzvetkov NT, Atanasov AG. 2020. The ethnopharmacological literature: An analysis of the scientific landscape. J Ethnopharmacol 250: 112414. 10.1016/j.jep.2019.112414. 